\title{
CONCEPTUAL MODEL OF COMPUTERIZED NATIONWIDE SCALE MULTIPARAMETER MONITORING SYSTEM FOR DRINKING WATER QUALITY
}

\author{
Anca Atanasescu1, Paul Nicolae Ancuţa ${ }^{2}$, Sorin Sorea ${ }^{3}$, \\ Constantin Anghel ${ }^{4}$, Dănut-Iulian Stanciu ${ }^{5}$ \\ 1,2,3,4,5 National Institute of Research and Development \\ in Mechatronics and Measurement Technique \\ Bucharest, Sos. Pantelimon 6-8, Romania \\ incdmtm@incdmtm.ro
}

\begin{abstract}
The article presents a multi-criteria functional model developed to analyze physicochemical and microbiological tests of drinking water for real-time monitoring. The model components comprise software and hardware units designed to collect real-time measurement, data storage, transmission and visualization for alphanumerical data of physical, chemical, and microbiological parameters of drinking water. The article will present a new concept useful in treating accidental water contamination: the ability to make determinations of physicochemical parameters using a portable electronic module portable, energetically autonomous, placed "on the spot", which wirelessly transmits data to the water operator's server, and hence to the national authority server. Data will be displayed in real time as a graphical evolution in time of decontamination. This functional model will serve the implementation, with low costs, of computer technology at local and national level for water quality controlling. The development of an information technology improves the methods of assessing water quality by using monitoring results as a basis for risk management actions.
\end{abstract}

Keywords: Multi Parameter Acquisition, Information Technology, Microbiology, First Section.

\section{Introduction}

European organizations involved in updating regulations concerning water management (WHO, OECD) [1], [2], [3] insisted on the need to improve the evaluation methods and management of microbiological safety of drinking water.

They suggest switching from using monitoring as a tool for checking the finished product towards the concept of using monitoring results as a starting point for risk management actions. Development of an information technology for microbiological characterization of the drinking water can fulfil the needs of improving water quality assessment methods, needs that have been increasingly referenced from 2011.

The proposed functional model envisages two directions of development:

1. It will create a nationwide computer network, accessible through the Internet, having as nodes the operators of drinking water and a national coordinating centre.

2. The model will implement a new concept useful in treating microbiological accidental contamination by offering the possibility to perform measurements of microbiological (type of pathogenic bacteria, with high risk to human health), physical and chemical parameters using a portable electronic module, energy autonomous, placed "on the spot", which can wirelessly transmit data to the drinking water distribution operators' servers.

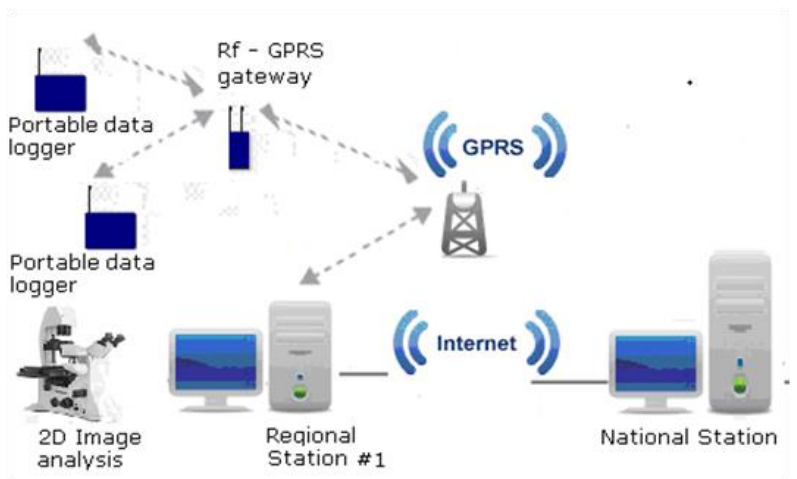

Figure 1: Architecture of Conceptual Model

The architecture of the conceptual model is shown in Fig. 1 and implements the following:

- methods for download data: physical, chemical and microbiological values are taken either from 
stations located in fixed control (for current measurements) or from mobile stations located in disaster locations

- methods for viewing data: physical, chemical and microbiological values can be viewed, as tables and reports, by any operator or customer of the water service through the dedicated website

- methods of transmitting data: physical, chemical and microbiological values are transmitted wirelessly via GPRS gateway to the local server (local water operator) and from there via the Internet by national server.

These conceptual model as stated above could be an alternative way to replace the conventional methods that use men force to monitor the environment and improves the performance, robustness, and provides efficiency in the monitoring system.

\section{Model Description}

The model consists of several sub-models which will be described as follows.

\subsection{Image Processing Model}

Regarding the information contained in an image, there are basically two disjoint areas: background and foreground.
They are delimited by partitioning criteria which determines a threshold. Image analysis is performed mainly on the foreground area. A particle can be defined as a region of pixels connected to each other by various criteria, belonging to the fore-ground area. Particle analysis involves processing operations using an algorithm that will produce meaningful results. INCDMTM team deals with image processing during several projects and activities [4] [5] [6] [7], [8], [9]

We have taken into consideration National Instruments NI Vision software and paradigm. NI IMAQ Vision is a software tools having more than 200 functions that provides either highly interactive operator actions or low level image processing functions whose union ensures the development of scientific and industrial applications in image processing.

Beside intrinsic LabView environment, IMAQ Vision functions can be used with various software development languages, namely Visual Basic, C, C\# and $\mathrm{C}++$.

Vision Assistant is a very useful software tool for software prototyping and preliminary testing stages. It creates a script with sequence of functions and associated parameters that have been selected by the user, in form of a pipeline processing. This script can then be later used by developing multiple scenarios and strategies using different images of the same category for algorithm validation.

An example of a core functional model in image vision is depicted in Figure 2.

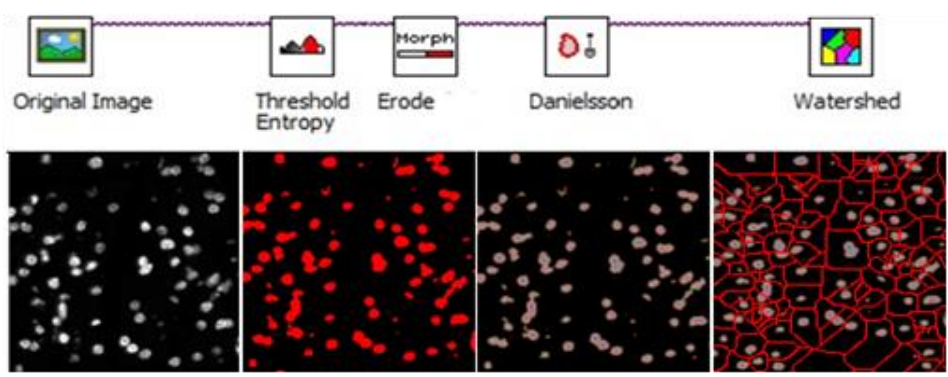

Figure 2: Image Processing Pipeline

The initial image is binarized using entropy criteria. Particles are slightly delimited using morphological erosion. Then a metric is defined for binary image using Danielsson approach which further delimitates particles and counts particles using Watershed algorithm.

A software package will be developed for analysing the microbiologic image of water sample, which will integrate the IMAQ Vision function.

\subsection{Portable Metering Model}

INCDMTM team deals with designing and manufacturing a complex solution for a multicriteria functional model in order to analyze physico- chemical and microbiological tests of drinking water for real-time monitoring.

The model components comprise software and hardware units designed to collect real-time measurement, data storage, transmission and visualization for alphanumerical data of physical, chemical and microbiological parameters of drinking water.

These model components are:

- an electronic device capable of acquiring data from a multi-parameter sensor (or from more sensors) having also two types of connectivity: local connectivity (PC desktop laptop) via RS232 interface and remote connectivity ( $\mathrm{RF}$ and a Gateway to GPRS). 
- a software component to integrate the device into a Wireless Sensor Network (WSN) at regional or national level.

- a management software unit to Monitor, Control and Management the data (alpha-numeric and image type data) running on local PC for tests.

\section{- MCU Implementation}

As in other INCDMTM paper [10] the implemented solution can use a microcontroller from Texas Instruments (TI) low power family MSP430.

MSP43F5529 could be our choice because of its features as follows:

- it is a 16-bit MCU with $128 \mathrm{~KB}$ Flash, 8KB RAM, up to $25 \mathrm{MHz}$ CPU speed, integrates USB 2.0 PHY timers

-it has 12-bit analogue-to-digital converter (ADC) with max. 16 channels (12 external channels and 4 internal ones).

- it has a very low-power consumption (only 4.2 nW per instruction, typical)

-it has multiple serial communication possibilities (UART, I2C, SPI)

The MSP4305529 based device will be hardware capable to acquire signals from various sources - analogue and digital - and to implement the processing functions needed for developing portable equipment by software. Data will be collected, analysed and transmitted to a proper serial transmitter, in order to integrate the device a wireless sensor network.

We have to mention that TI provides a system of support such as development tools, technical documentation, reference designs, application samples, customer support and services.

\section{- Smart Probes and Sensors}

For measuring the water parameters, the hardware device will acquire data from the tested water by the means of a large range of digital or analogue physicochemical sensors. These sensors usually integrate an electronic part that pre-process and transmits data to any controllers fitted with digital (RS-485) or analogue (current or volt-age) inputs.

Although more expensive, digital sensors are preferable because of their features: they are compact, robust and waterproof in order to be adaptable to any fields or applications, either handheld or fixed units. Furthermore, their conception allows a very low-power consumption and are disruption-resistant.

The digital and analogue inputs offered by the electronic design enables virtually any parameter to be measured and logged by connecting an appropriate sensor. Parameters that can be measured and logged are:
- $\mathrm{pH}$

- Dissolved oxygen (optical or electrochemical)

- ORP

- Conductivity

- Temperature

- Turbidity, etc.

In references [11-14] there area few examples of extremely versatile digital probes, allowing the user to automatically configure it with different sensor for different applications in minutes.

- Wireless implementation

The proposed model develops an electronic system with the capability of short-range and longrange communications for integration into a Wireless Sensor Network.

Today's industries offer many commercial offthe-shelf cost-effective, low-power solutions for short range, long range, mesh and IP networks, personal area networks and more.

The challenge is to find a competitive solution designed to simplify wireless development and certification by minimizing the amount of RF expertise.

INCDMTM started the development of the transmission module (hardware and software) with different communications options (wired \& wireless) so it can get adapted to different situations.

Different communications options have been carried out - for short range communications have been considered and analysed: IEE 802.15.4 based (Zigbee, 6LowPAN, Wireless M-BUS), WI-FI, Bluetooth LE and others solutions.

At this stage, the chosen solution for wireless will be a RF module, integrated into the device. Long range implementation is based on a 'Out of shelves' Gatewaywith GSM/GPRS capabilities. It functions like a gateway from $\mathrm{RF}$ frequencies to $3 \mathrm{G} / 4 \mathrm{G}$ frequencies. It is mainly designed for transparent data transmission.

The gateway connects the designed electronic device with a short-range RF inter-face via mobile communication to internet. The big advantage of this solution is the integration of a low-cost RF module instead of integrating a pricey GPRS modem in every measuring device. Besides saving costs, the gateway can compensate the poor connectivity of GPRS in many cellars.

The result has to satisfy all EU country regulations, remote locations and the various desired applications.

\subsection{Data Server Model}

The data management system (Figure 3) is designed as a client-server application where the client application is placed at measuring physical and biological parameters centre (network nodes) and the application server is implementing in a central server. 


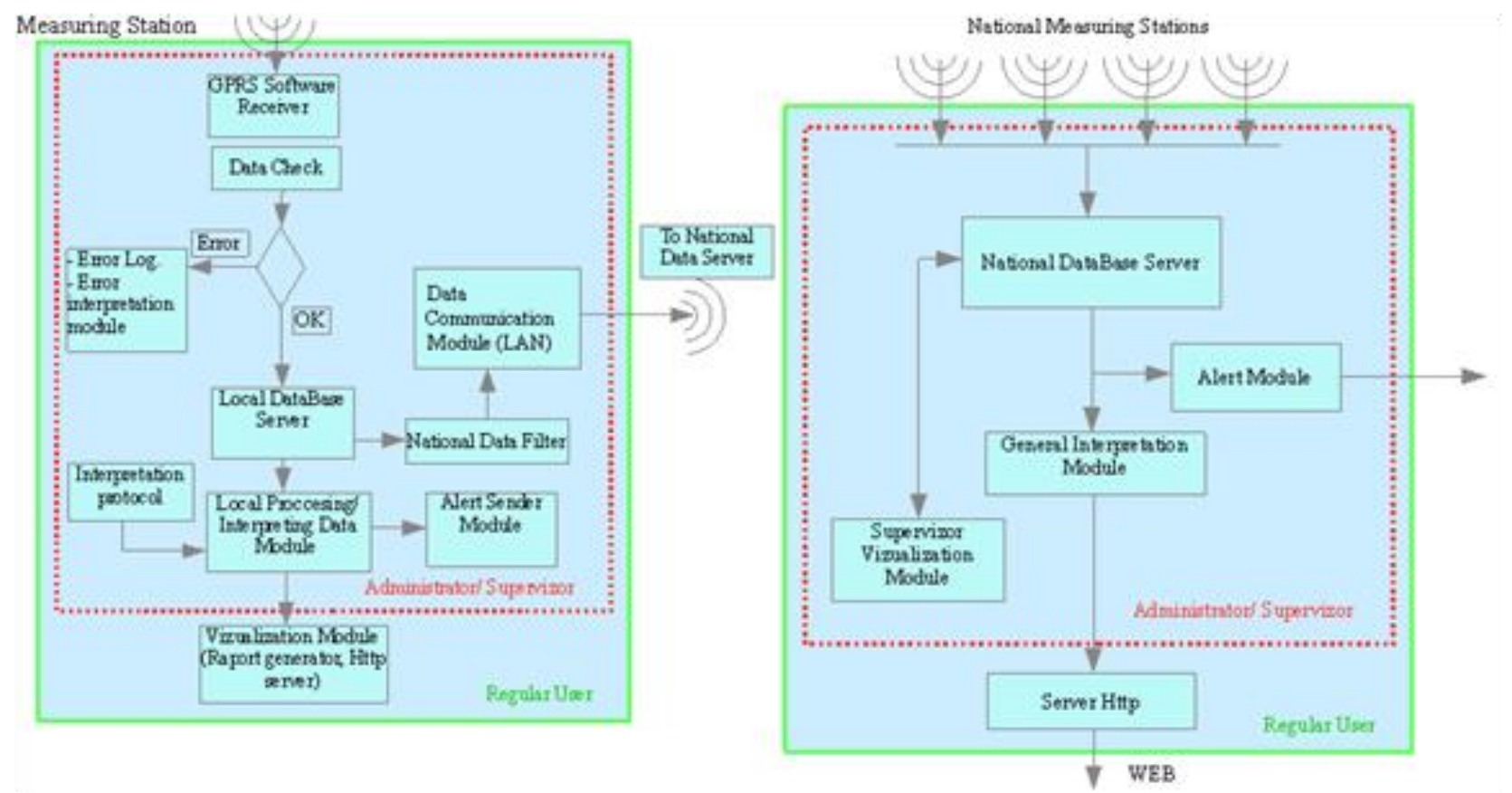

Figure 3: Data Management System

Each of these components performs dedicated functions as follows:

- The measuring stations do the following activities:

- define (and change) algorithms the raw metering values processing

- locally store the measurements value transmitted by the portable metering unit both as raw values as well as processed values (according to current processing algorithm)

- create local reports according to specifications

- define what kind of data is to be sent to the central unit

- exchange data with central server.

- The central server does the following activities:

- stores measurement data received from client stations

- define general security rules required by law

- validates and interprets data within the implemented limits and security rules

- defines and issues security alerts as defined by security rules

- enables owner defined structures and alert's values for enrolled users

- generates and issues regular reports

- enables instant access to measurement data by presenting data in an HTTP server (site); site access is defined using security settings;

\section{Conclusion and Future Works}

The article presents a conceptual model design to analyze physicochemical and microbiological tests of drinking water for real-time monitoring.

The model components comprise software and hardware units designed to collect real-time measurement, data storage, transmission and visualization for alphanumerical data of physical or chemical parameters and for image data of microbiological parameters.

The future work will imply the implementation of the presented solutions and finally of testing it on an environmental monitoring system. This system must have a low power consumption, low cost and to be a convenient way for real-time monitoring of the specific parameters. For flexibility and easy future updates, we will choose a modular architecture with interface compatibility.

The development effort will focus on the improvements required to adapt existing technology to the physical demands. In order to maximize the long-term viability of the system, commercial-offthe-shelf technology will be utilized whenever possible.

\section{Acknowledgment}

This work was supported by a grant of the Romanian National Authority for Scientific Research and Innovation CNCS/CCCDI-UEFISCDI, project number PN-III-P2-2.1-PED-2016-0965 within PNCDI III. 


\section{References}

[1] WHO's Guidelines for Drinking-water Quality [WHO.(1993)-second edition and WHO. (2004)third edition].

[2] OECD, WHO - Assessing Microbial Safety of Drinking Water, IWA Publishing(2003).

[3] WHO, Development of a Strategy on Water Quality and Health.2012.

[4]http://www.who.int/water_sanitation_health/dw $\mathrm{q} / \mathrm{en} /$

[5] Atanasescu, A., Sorea, S., Ancuta, P.N.: Modern method and reference database for the metallographic determination of the microstructure of the sintered hard metals according to the EU quality standards. Romanian Review Precision Mechanics, Optics and Mechatronics ISSN: 1584-5982, Nb.28/2005, pp.522...525; http://www.incdmtm.ro/editura/documente/p ag.\%20522-525.\%20Articol_XV.pdf.

[6] *** Research on using the modern systems for metallographic determination of microstructure and porosity for parts made of sintered metal carbides. R\&D Project, Contract RELAPSING 982/2003.

[7] Mica, C.A., Ancuţa, P.N.: Techniques used in medical 2D images for contour detection, Review of Romanian Society of Mechatronics SROMECA (2009)

[8] Ancuţa, P.N.: Contribution to virtual emulation of implant surgery in human lower limbs" Ph.D. Thesys, pages 24.29 and 37. 40 UPB, Faculty of Mechanical Engineering and Mechatronics.
[9] Sorea, S., Atanasescu, A., Ancuţa, P.N., Manescu, H.Al.: NonContact Measurement of Thread's Holes Using Video Systems. ICNAAM - RhodosGrecia, (22-28 sept 2014), Dependable Mechatronic Systems - Proceedings of the International Conference on Numerical Analysis and Applied Mathematics 2014 (ICNAAM2014).

[10] Constantinescu, Al., Atanasescu, A., Sorea, S., Anghel, C.: Modular optoelectronic systems used in transport logistics flows automatic industrial production, MECAHITECH 2015, Bucharest, Romania (2015).

[11] Sorea, S., Atanasescu, A., Ancuţa, P.N., Anghel, C., Gheorghe, Gh.: Parameters Monitoring Module for Wireless Sensor Network - a WLAN-based Approach, 8th International Conference on Innovations, Recent Trends and Challenges in Mechatronics, Mechanical Engineering and New High-Tech Products Development, MECAHITECH'16 International Conference, Bucharest, Romania, September 8th-9th, (2016).

[12] https://www.ysi.com/exo1

[13] http://www.ott.com/products/water-quality2 /hydrolab-ds5x-multiparameter-data-sonde855/

[14] http://www.rshydro.co.uk/water-qualitymonitoring-equipment/water-quality-testingequipment/multiparameter-water-qualitysonde/manta-2-multi-parameter-waterquality-sonde/

[15] https://in-situ.com/products/water-qualitytesting-equipment/aqua-troll-600multiparameter-sonde/ 